\title{
EQUIVARIANT CLASSIFYING SPACES AND FIBRATIONS BY
}

\author{
STEFAN WANER
}

\begin{abstract}
Explicit classifying spaces for equivariant fibrations are constructed using the geometric two-sided bar construction. The constructions are then extended to classify stable equivariant spherical fibrations and equivariant $K$-theory. The ambient groups is assumed compact Lie.
\end{abstract}

In order to be able to prove an equivariant version of the Adams Conjecture [Wa1], it is certainly helpful to have a classifying space for equivariant stable spherical fibrations, and to prove that they lead to a generalized equivariant cohomology theory [MHW].

Stasheff first constructed classifying spaces for various categories of fibrations in [St1], and these have proved to be an indispensable tool for homotopy theorists. The purpose of this paper is to construct explicit classifying spaces for various categories of stable and unstable equivariant fibrations over suitable base spaces. This will be done using a generalized "classifying space machine" based largely on that of Peter May in Classifying spaces and fibrations [Ma1]. As a by-product, we shall also obtain explicit classifying spaces for the various categories of (stable and unstable) equivariant bundles, thereby providing alternate models of spaces constructed by R. Lashof and M. Rothenberg in [La1], as well as new versions of the classifying spaces for equivariant $K$-theory. (See, for example, [Mo1].)

In order to be able to construct universal $G$-fibrations and to prove a classification theorem, the foundations of $G$-homotopy theory and $G$-cellular theory must be put in order. This is done in [Wa2] for $G$-compact Lie, and will enable us to prove our classification theorem with the full generality of $G$-compact Lie. The foundational theory of $G$-fibrations is discussed in [Wa3] and will be referred to here as needed.

The technique of our approach to the classification will be to restrain the fibers to lie in an appropriate "category of fibers" which (usually) contains a prototype space $F$ with varying actions of closed subgroups of $G . F$ serves as a homotopy model for the fibers of a given fibraton. The concept of a $G$-fibration with fiber $F$ [Wa3] may serve as a motivating example. (Without such a prototype, the family of classes of equivariant fibrations over a point are too large to be a set.) When $F$ is compact, we can classify up to strict fiberwise $G$-homotopy, and when $F$ is not compact, we are still able to classify up to weak fiberwise $G$-homotopy equivalence.

Received by the editors September 7, 1978 and, in revised form, January 15, 1979.

AMS (MOS) subject classifications (1970). Primary 54H15.

Key words and phrases. Closed subgroup, classifying space, classify, category of fibers, equivariant fibrations, stable equivariant fibrations, bar constructions. 
I am greatly indebted to Peter May for his valuable suggestions, and to Dick Lashof and Mel Rothenberg for their encouragement and assistance. I also wish to thank H. Hauschild for his suggestions which helped lead to alternate formulations of the classifying spaces for stable equivariant spherical fibrations and for equivariant $K$-theory.

\section{CONTENTS}

0. Notations, definitions and statement of main result

1. Equivariant fibrations and categories of fibers

1.1. Equivariant categories of fibers

1.2. Fibrations associated with an equivariant category of fibers

1.3. Principalization functors

2. Construction of equivariant classifying spaces

2.1. Equivariant simplicial spaces and realizations

2.2. Two-sided equivariant bar constructions

2.3. Proof of the main classification theorem

2.4. Comments on the universal base spaces

3. Based fibrations, bundles and stable fibrations

3.1. Based $G$-fibrations

3.2. Equivariant categories of bundle fibers

3.3. Stable $G$-fibrations

3.4. Some remarks on equivariant $K$-theory

3.5. Alternate models for certain classifying spaces

0. Notations, definitions and statement of main result. Throughout, $G$ will be assumed to a compact Lie group. $G \mathscr{Q}$ will denote the category of compactly generated weak Hausdorff spaces possessing a specified right $G$-action, and of continuous $G$-maps. The corresponding category of based spaces will be denoted by $G \mathcal{T}$ ( $G$ always fixed the basepoint), and the associated homotopy categories will be denoted by $h G \mathcal{Q}$ and $h G \mathcal{T}$.

In [Wa3], the concept of a $G$-fibration with fiber $F$ is introduced. Let $\delta F(X)$ denote the family of equivalence classes (up to $G$-fiberwise homotopy equivalence) of $G$-fibrations with compact fiber $F$ over a given "suitable" $G$-space $X$. (The notion of a "suitable" $G$-space will be made precise in $\S 3$, where the family $\mathcal{E} F(X)$ will also be discussed in more careful detail.) In effect, our classification theorem will read as follows:

Theorem 0. There exists a G-space B, unique up to G-homotopy, and a natural equivalence

$$
\Phi: \mathcal{E} F(X) \cong h G \mathscr{Q}(X, B) .
$$

Of course, it follows that $\mathcal{E} F(X)$ is a set.

\section{Equivariant fibrations and categories of fibers.}

1.1. Equivariant categories of fibers. Fibrations whose fibers are constrained to lie in a prespecified "category of fibers" are the natural generalization of fiber bundles (over suitable base spaces) and thus accessible to analysis in much the same way as 
bundles. May, in [Ma2], presents a comprehensive analysis of such fibrations. Here, we adapt this analysis to the equivariant case.

Definition 1.1.1. Let $F$ be a space in $\mathcal{Q}$, and let $\Lambda$ be a collection of right actions $\alpha$ of closed subgroups $H_{\alpha}$ on $F$ such that $\left\{H_{\alpha}\right\}$ is a complete set of representatives of conjugacy classes of closed subgroups of $G$. An equivariant category of fibers with distinguished fiber $F$ is a pair $(G \mathscr{F}, F)$ such that $G \mathscr{F}$ is a category each of whose objects is a map $p: P \rightarrow Q$ in $G \mathscr{U}$ with $Q$ a $G$-orbit, and each of whose morphisms is a pair of $G$-maps yielding a commutative square:

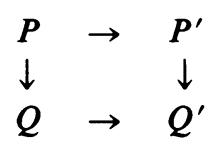

In addition, $G \mathscr{F}$ is required to have the following properties:

(i) For each $\alpha \in \Lambda$, the maps $p_{\alpha}: F_{\alpha} \times{ }_{H_{\alpha}} G \rightarrow G / H_{\alpha}$ are in $G \mathcal{F}$. (Here, $F_{\alpha}$ denotes $F$ with the right action $\alpha$ by $H_{\alpha}$.)

(ii) For each pair $\left(p: P \rightarrow Q, p^{\prime}: P^{\prime} \rightarrow Q^{\prime}\right)$ of objects in $G \mathscr{F}$, and for each morphism $(\bar{\theta}, \theta): p \rightarrow p^{\prime} \bar{\theta}$ restricts to a weak $G_{x}$-equivalence: $p^{-1}(x) \rightarrow p^{\prime-1}(G(x))$ for each $x \in Q$.

(iii) If $p: P \rightarrow Q$ is an object in $G \mathcal{F}$, and if $\theta: G / K \rightarrow Q$ is in $G \mathscr{Q}$, with $K=H_{\alpha}$ for some $\alpha \in \Lambda$, then there exists a morphism $(\bar{\theta}, \theta) p_{\beta} \rightarrow p$ for some $\beta \in \Lambda$.

(iv) Observing that $G \mathscr{F}$ is a topological category, we require the natural projections

$$
\pi_{\alpha \beta}: G \mathscr{F}\left(p_{\alpha}, p_{\beta}\right) \rightarrow \tilde{G} \mathscr{U}\left(G / H_{\alpha}, G / H_{\beta}\right)
$$

(where $\tilde{G} \mathscr{U}\left(G / H_{\alpha}, G / H_{\beta}\right) \subset G \mathscr{U}\left(G / H_{\alpha}, G / H_{\beta}\right)$ denotes the image of $\pi_{\alpha \beta}$, the existence of a map $G / H_{\alpha} \rightarrow G / H_{\beta}$ not implying in general that we can cover it by a map $(\bar{\theta}, \theta): p_{\alpha} \rightarrow p_{\beta}$ since we may have $H_{\alpha}=H_{\alpha^{\prime}}$ for two incomparable actions $\alpha$ and $\left.\alpha^{\prime}\right)$ to be quasifibrations such that if $\phi \in G \mathscr{F}\left(p_{\beta}, p_{\gamma}\right)$, then, in the commutative diagram:

$$
\begin{array}{ccc}
G \mathscr{F}\left(p_{\alpha}, p_{\beta}\right) & \stackrel{F}{\rightarrow} & G \mathscr{F}\left(p_{\alpha}, p_{\gamma}\right) \\
\pi_{\alpha \beta} \downarrow & & \downarrow \pi_{\alpha \gamma} \\
G \mathscr{Q}\left(G / H_{\alpha}, G / H_{\beta}\right) & \stackrel{f}{\rightarrow} & G \mathscr{U}\left(G / H_{\alpha}, G / H_{\gamma}\right)
\end{array}
$$

induced by $\phi, F$ restricts to a weak equivalence on each fiber.

(v) If $p: P \rightarrow Q$ is in $G \mathscr{F}$, then so is $p \times 1_{t}: P \times\{t\} \rightarrow Q \times\{t\}$ for each $t$ in $I$. Similarly, if $\Theta: p \rightarrow q$ is in $G \mathscr{F}$, then so is $\Theta \times 1_{t}: p \times 1, \rightarrow q \times 1$, for each $t \in I$.

Observe that the full subcategory of $G \mathscr{F}$ whose objects are the $p_{\alpha}$ for $\alpha \in \Lambda$ is a small topological category (the set $\left\{p_{\alpha}: \alpha \in \Lambda\right\}$ of objects being given the discrete topology). Our classifying spaces will take the form of categorical constructions based on this subcategory of $G \mathscr{F}$.

EXAMPLe 1.1.2. As usual, $G$ will be a compact Lie group.

(a) Let $F$ be a space, and let $\Lambda$ be a set of actions $\alpha$ of closed subgroups $H_{\alpha}$ on $F$ such that:

(i) $\left\{H_{\alpha}\right\}$ gives a complete set of representatives of $G$-orbit types. 
(ii) The collection of actions $\left\{\alpha \in \Lambda: H_{\alpha}=H\right\}$ is a complete representative set of $H$-homotopy classes of actions by $H$ on $F$ such that $F \in H$ W.

Define a category of fibers $(G \mathscr{F} \mathscr{Q}, F)$ whose objects are all $G$-maps $p: P \rightarrow Q$ such that there is a fiberwise equivariant weak equivalence ${ }^{1}(\theta, \bar{\theta}): p_{\alpha} \rightarrow p$ for some $G$-homeomorphism $\bar{\theta}: G / H_{\alpha} \rightarrow Q$ and some $\alpha \in \Lambda$. The morphisms of $G \mathscr{F} \mathscr{U}$ are all pairs $(\bar{\theta}, \theta)$ which satisfy $1.1 .1($ ii).

(b) Let $(G \mathscr{F} \mathscr{W}, F)$ be specified such that $G \mathscr{F} \mathscr{W}$ is the full subcategory of $G \mathscr{F} \mathscr{Q}$ obtained by replacing "weak equivalence" by "equivalence" everywhere in the definition above.

Note that, when $F$ is compact, the set $\Lambda$ may be assumed countable.

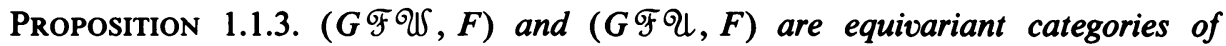
fibers.

Proof. Conditions (i) and (ii) of 1.1.1 are easily seen to be met, and (v) is trivial.

To establish (iii), suppose given a $G$-map $\theta: G / K \rightarrow Q$ for an object $p: P \rightarrow Q$ in $G \mathscr{F} \mathcal{Q}$ (or $G \mathscr{F} \mathscr{W}$ ). We may as well assume that $p=p_{\alpha}$ for some $\alpha \in \Lambda$, by definition of the category $G \mathscr{F} \mathscr{U}$. Now there is a $G$-homeomorphism $\phi$ making the following diagram commutative:

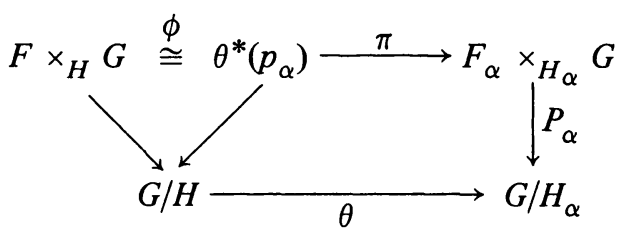

where the action of $H$ on $F$ is defined by

$$
f \circ h=f \circ \alpha\left(h^{a}\right),
$$

where $a \in G$ is any element such that $\theta$ is left multiplication (of right cosets) by a (any $G$-map $G / H \rightarrow G / H$ being of this form), and $h^{a}=a^{-1} h a$. Since $\pi$ restricts to an $H$-equivariant homeomorphism over $\mathrm{He} \in \mathrm{G} / \mathrm{H}$, we have shown (iii).

1.1.1(iv) is a formal consequence of the fact that $p$ is an equivariant fibration for each $\alpha \in \Lambda$ (see [La1]); local triviality of $p$ follows from the existence of local sections of $G \rightarrow G / H_{\alpha}$. Indeed, the maps $\pi_{\alpha \beta}: G \mathscr{F}\left(p_{\alpha}, p_{\beta} \rightarrow G \mathscr{Q}\left(G / H_{\alpha}, G / H_{\beta}\right)\right)$ are fibrations.

1.2. $G \mathcal{F}$ spaces and $G \mathcal{F}$ fibrations. Recall the notion of a $G$-space $p: E \rightarrow B$ over $B$ from [Wa3]. (This is similar to James' category of spaces over a fixed space $B$.)

Definition 1.2.1. Let $(G \mathscr{F}, F)$ be an equivariant category of fibers. A $G \mathscr{F}$ space is a $G$ space $p: E \rightarrow B$ such that $p \mid: p^{-1}(Q) \rightarrow Q$ is in $G \mathscr{F}$ for each orbit $Q$ in $B$. A morphism of $G \mathscr{F}$ spaces is a morphism of $G$ spaces which restricts to a morphism in $G \mathscr{F}$ over each orbit. We thus have a category of $G \mathcal{F}$ spaces and an associated homotopy category. A $G \mathscr{F}$-quasifibration is a $G \mathscr{F}$ space which is also a $G$-quasifibration [Wa3].

\footnotetext{
${ }^{1}$ With respect to the action of the appropriate isotropy subgroup.
} 
Definition 1.2.2. A $G \mathcal{F}$ space $p: E \rightarrow B$ is said to be a $G \mathscr{F}$ fibration if it satisfies the $G \mathscr{F} C H P$, defined by the following diagram of morphisms of $G \mathscr{F}$ spaces:

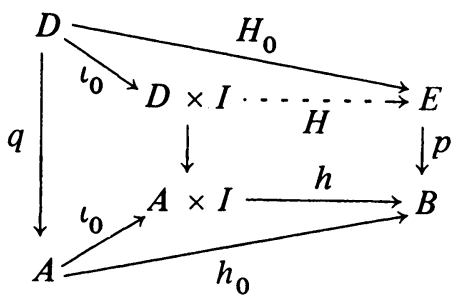

in which $q$ and $p$ are given $G \mathcal{F}$ spaces, $h$ is in $G \mathscr{U}$, and $\left(H_{0}, h_{0}\right)$ is a given morphism of $G \mathscr{F}$ spaces.

Notations 1.2.3. If $p: E \rightarrow B$ is in $G \mathcal{Q}$, denote by $\Gamma p: \Gamma E \rightarrow B$ the gammafication of $p$ discussed in [Ma2, \$3.2]. (Briefly, $\Gamma E \subset \pi B \times E$ is the set of pairs $(\lambda, e)$, where $\lambda$ is a Moore path in $B$ starting at $p(e)$, and $\Gamma p$ is the endpoint projection $\Gamma p(\lambda, e)=\lambda(l(\lambda))$, where $l(\lambda)$ is the length of $\lambda$; that is, $\lambda: \overline{\mathbf{R}}^{+} \rightarrow B$ is constant for parameters $>l(\lambda)$.) Observe that $\Gamma E$ inherits a natural $G$-action such that $\Gamma p$ is a $G$-map. We also have the obvious $G$-map $\eta: E \rightarrow \Gamma E$ over $B$, and the path addition map $\mu: \Gamma(\Gamma E) \rightarrow \Gamma E$ over $B$. These maps are discussed in detail in [Ma2, §3.2].

DEFINITION 1.2.4. Let $p: E \rightarrow B$ be a $G \mathscr{F}$ space for some category of fibers $(G \mathscr{F}, F)$. A $G \mathscr{F}$ lifting map for $p$ is a $G$-map $\xi: \Gamma E \rightarrow E$ over $B$ such that $\xi \circ \eta=1$, and such that the map

$$
\xi \mid: \pi^{-1}(\beta . G) \rightarrow p^{-1}(\beta(\infty) \cdot G)
$$

is in $G \mathscr{F}$ for each $\beta \in \pi B$, where

$$
\pi: \Gamma E \rightarrow \pi B
$$

is the natural map. (This condition is equivalent to that in [Ma2, \$3.2] in the nonequivariant case.)

The following proposition displays the universality of the gamma construction just mentioned:

Proposition 1.2.5. A G F space $p: E \rightarrow B$ is $a G \mathcal{F}$ fibration if and only if it has a $G \mathscr{F}$ lift $\xi$.

The proof of [Ma2, \$3.4] goes over to this equivariant case verbatim. Observe that, in this general setting, the above proposition provides a clue as to what is entailed by equivalence of two $G \mathscr{F}$ fiberings (see 2.3.5).

We now examine the behavior of the $\Gamma$ function on categories of fibers:

Definition 1.2.6. A category of fibers $(G \mathscr{F}, F)$ is $\Gamma$-complete in a full subcategory $G \mathcal{Q}$ of $G \mathscr{Q}$ if the following statements are true for $G \mathcal{F}$ quasifiberings $p$ : $E \rightarrow B$ with $B$ and $E$ in $G \mathcal{Q}$ :

(i) $\Gamma p$ is a $G \mathscr{F}$ fibration with $G \mathscr{F}$-lifting function $\xi$;

(ii) $\eta: E \rightarrow \Gamma E$ is a $G \mathscr{F}$ map over $B$;

(iii) $\Gamma$ takes $G \mathscr{F}$ maps between quasifibrations to $G \mathscr{F}$ maps. 
Proposition 1.2.7. The categories $(G \mathscr{F} \mathcal{W}, F)$ and $(G \mathscr{F} \mathscr{W}, F)$ are $\Gamma$-complete in $G \mathcal{Q}$ and $G$ ข respectively.

Proof. For $G \mathscr{F} \mathscr{Q}$, (i) and (ii) are immediate, since the five-lemma implies that $\mu$ is a $G \mathscr{F} \mathscr{Q}$ lift.

The proof for $G \mho$ requires the following result, which is proved in [Wa2] and [Wa3]: If $p: E \rightarrow B$ is in $G \mathscr{W}$, then the homotopy-theoretic fiber is of the $G$-homotopy type of a based $G-C W$ complex. Property (iii) now follows by an easy argument.

REMARK 1.2.8. Observe that the proof of 1.2 .5 shows that a $G \mathscr{F}$ fibration is a $G$-fibration whatever the choice of category of fibers $(G \mathscr{F}, F)$. The converse is not always true, although every $G$-fibration with fiber $F$ (cf. [Wa3]) is a $(G \mathscr{F} \mathscr{W}, F)$ fibration by definition. The following proposition tells us when a $G$-fibration is a $(G \mathscr{F}, F)$ fibration.

Proposition 1.2.9. Suppose that $p: E \rightarrow B$ is a $G$-fibration such that the restrictions of $p$ to the preimages of a set $\left\{Q_{\alpha}\right\}$ of orbits in $B$ are $G \mathscr{F} \mathcal{Q}$-spaces, and that the inclusion $\amalg Q_{\alpha} \rightarrow B$ induces a surjection $\pi_{0}^{H}(\iota): \pi_{0}^{H}\left(\amalg Q_{\alpha}\right) \rightarrow \pi_{0}^{H}(B)$ for each closed subgroup $H$. Then $p$ is $a G \mathcal{F} \mathscr{U}$ fibration.

Proof. This is a formal consequence of the $G$-CHP.

Corollary 1.2.10. Let $p: E \rightarrow B$ be $a G$-fibration such that there is a point $q$ in each component of each fixed point set of $B$ with a (weak) $G_{q}$-equivalence $F_{\alpha} \rightarrow$ $p^{-1}(q)$ for some action $\alpha$ of $G_{q}$ on $F$ such that $F_{\alpha}$ has the $G_{q}$-homotopy type of a $G_{q}-\mathrm{CW}$ complex. Then $p$ is $a G \mathscr{F} \mathscr{W}(G \mathscr{F} \mathscr{U})$ fibration.

Thus, for example, all $G$-bundles which satisfy the $G$-CHP are $G \mathscr{F} \mathscr{W}$ fibrations for some fiber $F$. ( $G$-bundles will be discussed in \$3.)

It is now apparent why the notion of a category of fibers is essential to an analysis of $G$-fibrations. Indeed, it is easy to see that the collection of $G$-equivalence classes of $G$-fiberings with contractible fiber over a point is too large to be a set, and hence is unclassifiable whenever $G \neq 1$.

Proposition 1.2.11. Suppose that B has a numerable G-cover by open tubes which deform equivariantly to specified "central" orbits. Then any $(G \mathscr{F} \mathscr{W}, F)$ map of $(G \mathscr{F} \mathscr{W}, F)$ fibrations over $B$ is $a G$-fiberwise homotopy equivalence.

Proof. This is immediate from the definition of the category $(G \mathscr{F} \mathscr{W}, F)$ and from the $G$-Dold Theorem [Wa3].

The forgetful functors also behave correctly:

Proposition 1.2.12. $\mathscr{F}_{H}: G \mathscr{U} \rightarrow H \mathcal{H}$ sends $(G \mathscr{F}, F)$ (quasi)fibrations to $(H \mathscr{F}, F)$ (quasi)fibrations for $G \mathscr{F}=G \mathscr{F} \mathscr{U}$ or $G \mathscr{F} \mathscr{W}$.

Proof. First, $(G \mathscr{F}, F)$ spaces go to $(H \mathscr{F}, F)$ spaces because $H$-maps $H / H^{\prime} \rightarrow X$ arise from $G$-maps $G / H^{\prime} \rightarrow X$ (consider fixed point sets).

Second, if $p: E \rightarrow B$ is a $G \mathscr{F}$ fibration, we may convert an $H \mathscr{F}$ lifting problem to a $G \mathscr{F}$ lifting problem by considering the following diagram: 


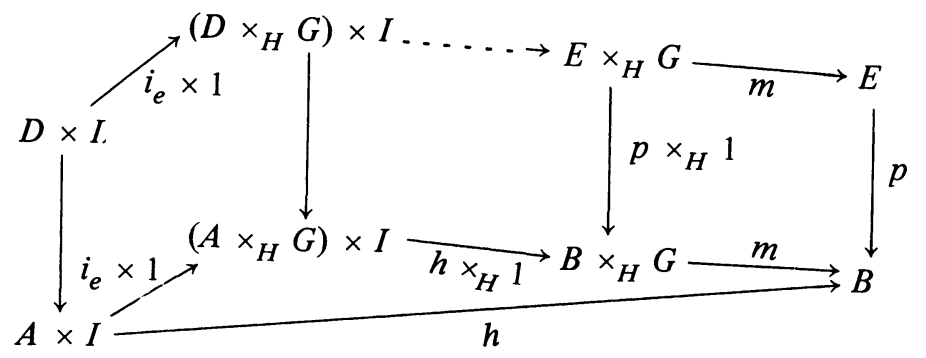

where all the squares are in $G$ except for the left-hand one, which is in $H$, and $m$ is obtained from the $G$-action, while $i_{e}$ is inclusion $a \mapsto(a, e)$.

(This can also be proved using the $G$-gamma construction.)

1.3. Principalization functors. We turn now to the formulation of equivariant principalization functors to enable us to construct the classifying maps in $\$ 2$.

Definition 1.3.1. Let $(G \mathscr{F}, F)$ be an equivariant category of fibers, and let $\Lambda$ denote its set of actions on $F$. For a $G \mathscr{F}$ space $p: E \rightarrow B$ and an $\alpha \in \Lambda$, define a map $P(p)_{\alpha}: P(E)_{\alpha} \rightarrow P(B)_{\alpha}$ in $\mathcal{Q}$ by setting $P(E)_{\alpha}=G \mathscr{F}\left(p_{\alpha}, p\right)$ and letting $P(B)_{\alpha} \subset G \mathcal{Q}\left(G / H_{\alpha}, B\right)$ be the image space obtained by passage to orbit maps. $P(p)_{\alpha}$ is the quotient map. (Recall that $p_{\alpha}: F_{\alpha} \times{ }_{H_{\alpha}} G \rightarrow G / H_{\alpha}$ is the quotient map and that all these notations were described in Definition 1.1.1.)

Definition 1.3.2. By a (nonequivariant) category of fibers, we shall mean a $\{1\}$-category of fibers (as in [Ma2]). If $(G \mathscr{F}, F)$ is a $G$-category of fibers with set of actions $\Lambda$, we define an associated category of fibers $\left(\mathscr{F}_{\alpha}, F_{\alpha}\right)$ for each $\alpha \in \Lambda$ as follows: $\mathscr{F}_{\alpha}$ is the category whose objects are the preimages of all points $q \in Q$, as $Q$ ranges through the codomains of objects $p$ in $G \mathcal{F}$, such that there exists a $G \mathcal{F}$ map $\Theta=(\bar{\theta}, \theta): p_{\alpha} \rightarrow p$ with $\theta([e])=q$. The morphisms in $\mathscr{F}_{\alpha}$ are then restrictions of those in $G \mathscr{F}$. It is easily verified that all the requirements for a category of fibers are met.

Let $\left(\mathbb{Q}_{\alpha}, A_{\alpha}\right)$ denote the associated principal category of fibers, as defined in [Ma2, §4.3]. (Briefly, $A_{\alpha}=\mathscr{F}_{\alpha}\left(F_{\alpha}, F_{\alpha}\right)$, and the objects of $Q_{\alpha}$ are of the form $\mathscr{F}_{\alpha}\left(F_{\alpha}, X\right)$ for $\left.X \in \mathscr{F}_{\alpha}.\right)$

Proposition 1.3.3. The operations $P(-)_{\alpha}$ define functors: $(G \mathscr{F}, F) \rightarrow\left(\mathbb{Q}_{\alpha}, A_{\alpha}\right)$ for each $\alpha$. Moreover, $P(-)_{\alpha}$ restricts to a functor from $G \mathcal{F}$ fibrations to $\mathcal{Q}_{\alpha}$ fibrations.

Proof. The first statement follows formally from the definitions. Let $p_{\alpha}:\left(\mathscr{F}_{\alpha}, F_{\alpha}\right)$ $\rightarrow\left(\mathbb{Q}_{\alpha}, A_{\alpha}\right)$ denote the principalization functor discussed in [Ma2, §4.3]. (Briefly, $P_{\alpha}(X)=\mathscr{F}_{\alpha}\left(F_{\alpha}, X\right)$ and $P_{\alpha}(f)=\mathscr{F}_{\alpha}\left(F_{\alpha}, f\right)$.) Then we have the following commutative diagram:

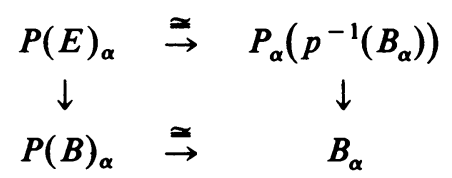

where $B_{\alpha} \subset B$ is the subspace $\left\{\theta([e]): \Theta=(\bar{\theta}, \theta): p_{\alpha} \rightarrow p\right\}$, the bottom homeomorphism is the correspondence $\theta \leftrightarrow \theta([e])$, and the top homeomorphism is

$$
(\bar{\theta}, \theta) \leftrightarrow \bar{\theta} \mid p_{\alpha}^{-1}[e] .
$$


Since the right-hand vertical arrow is an $\left(\mathbb{Q}_{\alpha}, A_{\alpha}\right)$ fibration by a purely formal argument [Ma2,\$4.5], the second assertion follows.

REMARK 1.3.4. We have the following amusing observation: If $(G \mathscr{F}, F)$ is a $G$-category of fibers with set of actions $\Lambda$, we may define nonequivariant categories of fibers $\mathscr{F}_{\alpha} \mathcal{U}$ and $\mathscr{F}_{\alpha} \mathscr{W}$ as in [Ma2, §6.2(iii)] by considering the $H_{\alpha}$ action on $F$. Then there are canonical equivalences:

$$
\tau_{\alpha}:\left(\mathcal{F} \mathcal{U}_{\alpha}, F_{\alpha}\right) \cong\left(\mathscr{F}_{\alpha} \mathcal{Q}, F_{\alpha}\right)
$$

and

$$
\tau_{\alpha}:\left(\mathscr{F} \mho_{\alpha}, F_{\alpha}\right)=\left(\mathscr{F}_{\alpha} W, F_{\alpha}\right)
$$

where $\mathscr{F} \mathscr{U}_{\alpha}$ and $\mathscr{F} \mathscr{W}_{\alpha}$ are as in Definition 1.3.2.

We now record the following information for later use:

Proposition 1.3.5. If $\theta_{1}$ and $\theta_{2}$ are G-homotopic maps $G / H_{\alpha} \rightarrow G / H_{\beta}$, and if $\theta_{1}$ is covered by a $G$-map $F_{\alpha} \times{ }_{H_{\alpha}} G \rightarrow F_{\beta} \times{ }_{H_{\beta}} G$, then so is $\theta_{2}$.

This is an immediate consequence of the G-CHP.

COROLlARY 1.3.6. If $B$ is the base space of $a G \mathcal{F}$ fibration, then for each $G$-map $\phi$ : $G / H \rightarrow B$, there is an action $\alpha \in \Lambda$ and an element $\tilde{\phi} \in P(B)_{\alpha}$ such that $\pi_{n}\left(P(B)_{\alpha}\right.$, $\tilde{\phi}) \cong \pi_{n}^{H}(B, \phi)\left(\right.$ where $\left.H_{\alpha}=H\right)$.

2. Construction of equivariant classifying spaces. Here, $G \mathscr{F}$ fibrations are classified. The main references for this chapter are [Ma2, \$§3 and 4].

2.1. Equivariant simplicial spaces and realization.

Definition 2.1.1. A $G$-simplicial space is a simplicial object in the category $G \mathscr{U}$ (see, for example, $[\mathbf{M a 3}, \S 1]$ for details). Since all the simplices $\Delta^{n}$ are in $G \mathscr{U}$ with trivial $G$-action, it follows that the geometric realization functor restricts to a functor from $G$-simplicial spaces to $G$-topological spaces, and all the functorial and homotopy properties continue to hold equivariantly (see [Ma4, §11]).

The important examples of $G$-simplicial spaces will be in the form of two-sided bar constructions. Other examples arise from Illman's equivariant singular homology and cohomology theories, ${ }^{2}$ but these will not be dealt with here (even though I am sure that our $G-C W$ theory can be used to construct an equivariant Serre spectral sequence).

2.2. Two-sided equivariant bar constructions. We recall some material from [Ma2, $\S 12$ ]. If $\Lambda$ is a discrete space (of objects), a $\Lambda$-graph is a space $\mathscr{B}$ (of arrows) together with continuous maps $S: \mathscr{B} \rightarrow \Lambda$ and $T: \mathscr{B} \rightarrow \Lambda$ (called source and target). A morphism of $\Lambda$ graphs is a continuous map $f: \mathscr{B} \rightarrow \mathscr{B}^{\prime}$ such that $S \circ f=S$ and $T \circ f=T$. If $\mathscr{B}$ and $\mathscr{B}^{\prime}$ are two $\Lambda$ graphs, let $\mathscr{B} \times{ }_{\Lambda} \mathscr{B}^{\prime}$ be the $\Lambda$-graph with arrow space $\left\{\left(b, b^{\prime}\right): S b=T b^{\prime}\right\} \subset \mathscr{B}^{\prime} \times \mathscr{B}^{\prime}$ and with $S\left(b, b^{\prime}\right)=$ $S\left(b^{\prime}\right)$ and $T\left(b, b^{\prime}\right)=T b$.

Define a right $\Lambda$-graph to be a space $\mathcal{O}$ with a map $S$ : $\mathcal{Y} \rightarrow \Lambda$; similarly, a left $\Lambda$-graph $\mathcal{X}$ has only $T: \mathscr{X} \rightarrow \Lambda$. We can then define products $\mathcal{Y} \times_{\Lambda} \mathscr{B}$ and $\mathscr{B} \times{ }_{\Lambda} \mathfrak{X}$ as right and left $\Lambda$-graphs, and $\mathcal{Y} \times_{\Lambda} \mathscr{X}$ as a space.

\footnotetext{
${ }^{2}$ See [II1].
} 
A monoid in the category of $\Lambda$-graphs is a triple $(\mathcal{Q}, C, I)$ where $\mathscr{Q}$ is a $\Lambda$-graph, and $C: \mathbb{Q} \times{ }_{\Lambda} \mathbb{Q} \rightarrow \mathbb{Q}$ and $I: \Lambda \rightarrow \mathbb{Q}$ are maps of $\Lambda$-graphs (called composition and identity) which satisfy the expected associativity and identity conditions. This is just a small topological category with object space $\Lambda$.

A right $\Lambda$-graph over $\mathscr{Q}$ is a right $\Lambda$-graph $\mathcal{O}$ together with a map $R$ : $\mathcal{Y} \times{ }_{\Lambda} \mathbb{Q} \rightarrow \mathcal{Y}$ of right $\Lambda$-graphs which satisfies $R\left(1 \times_{\Lambda} C\right)=\Re\left(\Re \times_{\Lambda} 1\right)$, and $\Re\left(1 \times{ }_{\Lambda} I\right)=\Re$.

Definition 2.2.1. If $\mathcal{Y}$ is a left $\lambda$-graph over a monoid $\mathscr{Q}$ such that $\mathscr{Y}$ also has a right $G$-action, which commutes with the source map $(S(y g)=S(y))$ and the $\mathcal{Q}$-action $(\Re(a, y g)=\mathscr{R}(a, y) g)$ then we say that $\mathcal{Y}$ is a left- $G$ - $\Lambda$-graph over $\mathcal{Q}$.

If $\mathcal{X}$ and $\mathcal{Y}$ are, respectively, right- $\Lambda$ - and left- $G$ - $\Lambda$-graphs over $\mathcal{Q}$, we may form the $G$-simplicial space $B_{*}(\mathcal{X}, \mathcal{Q}, \mathcal{Y})$ and its realization $B(\mathcal{X}, \mathcal{Q}, \mathcal{Y})=$ $\left|B_{*}(\mathcal{X}, \mathcal{Q}, \mathcal{Y})\right|$ in $G \mathscr{Q}$ by following [Ma2, §12].

EXAMPLE 2.2.2. Let $(G \mathscr{F}, F)$ denote a category of fibers, and let $\Lambda$ denote the discrete space of distinct actions of closed subgroups on $F$ associated with the category $G \mathscr{F}$. Define a monoid $\mathbb{Q}$ in the category of $\Lambda$-graphs by letting

$$
\mathcal{Q}=\amalg_{\alpha, \beta \in \Lambda} G \mathscr{F}\left(p_{\alpha}, p_{\beta}\right)
$$

with the natural source and target maps, and monoid structure given by composition in $G \mathscr{F}$. We have the following examples of $\Lambda$-graphs over $\mathcal{Q}$. (Henceforth, $\mathbb{Q}$ will always be as above.)

(i) $\mathcal{X}=\Lambda$ with action $\Lambda \times{ }_{\Lambda} \mathbb{Q} \rightarrow \Lambda$ the only possible one;

(ii) $\mathfrak{X}=\mathscr{P}(E)$, where $\mathscr{P}(E)=\amalg_{\alpha \in \Lambda} P(E)_{\alpha}$ and where the action $\mathscr{P}(E) \times{ }_{\Lambda} \mathscr{Q}$ $\rightarrow \mathscr{P}(E)$ is given by composition.

(iii) $\mathcal{Y}=\theta$, where $\theta=\amalg_{\alpha \in \Lambda} G / H_{\alpha}$ and where the action $\mathbb{Q} \times_{\Lambda} \theta \rightarrow \theta$ is given by $((\bar{\theta}, \theta),[g]) \rightarrow \theta([g])$.

(iv) $\mathcal{Y}=\mathscr{F}$, where $\mathscr{F}=\amalg_{\alpha \in \Lambda} F_{\alpha} \times_{H_{\alpha}} G$ and where the action $\mathcal{Q} \times_{\Lambda} \mathscr{F} \rightarrow \mathscr{F}$ is given by evaluation.

(v) $\mathcal{Y}=\mathbb{Q}_{\beta}$, where $\mathbb{Q}_{\beta}=\amalg_{\alpha \in \Lambda} G \mathscr{F}\left(p_{\beta}, p_{\alpha}\right)$ and where the action $\mathbb{Q} \times{ }_{\Lambda} \mathbb{Q}_{\beta} \rightarrow$ $Q_{\beta}$ is given by composition in $G \mathscr{F}$.

(vi) $\mathcal{Y}=\tilde{\mathbb{Q}}_{\beta}$, where $\tilde{\mathbb{Q}}_{\beta}=\amalg_{\alpha \in \Lambda} \pi_{\beta \alpha} G \mathscr{F}\left(p_{\beta}, p_{\alpha}\right)$ (see 2.2.1) and where the action $\mathbb{Q} \times{ }_{\Lambda} \tilde{\mathbb{Q}}_{\beta} \rightarrow \mathbb{Q}_{\beta}$ is given by $((\bar{\theta}, \theta), \phi) \rightarrow \theta \circ \phi$.

Here, the graphs $\mathscr{Q}_{\beta}$ and $\tilde{\mathscr{Q}}_{\beta}$ have trivial $G$-action, and are regarded as nonequivariant graphs.

Proposition 2.2.3. If $\mathbb{Q}$ is as above, and if $I(\alpha)$ is nondegenerate for each $\alpha \in \Lambda$, then:

(i) The maps $p: B(\mathcal{X}, \mathcal{Q}, \mathcal{F}) \rightarrow B(\mathcal{X}, \mathcal{Q}, \mathcal{O})$ are $G \mathscr{F}$ quasifibrations for arbitrary $\Lambda$-graphs $\mathcal{X}$ over $\mathbb{Q}$.

(ii) If $\mathscr{X}_{\alpha}=T^{-1}(\alpha) \subset \mathcal{X}$ is nonempty and aspherical for each $\alpha \in \Lambda$, then the map

$$
q: B(\mathcal{X}, \mathcal{Q}, \mathcal{Y}) \rightarrow B(\Lambda, \mathcal{Q}, \mathcal{Y})
$$

is an equivariant quasifibration, and is thus a weak G-equivalence.

(iii) The maps $p_{\alpha}: B\left(\mathcal{X}, \mathcal{Q}, \mathbb{Q}_{\alpha}\right) \rightarrow B\left(\mathcal{X}, \mathcal{Q}, \tilde{\mathbb{Q}}_{\alpha}\right)$ are $A_{\alpha} \mathscr{W}$ quasifibrations whenever $F$ is in $H_{\alpha}$ W for each $\alpha \in \Lambda$. 
The maps $p, q$ and $p_{\alpha}$ are the natural maps induced by projection on the simplicial space level.

Proof. (i) In order to adapt the argument in [Ma2, 87.6] using Definition 1.4.4, we observe that the $p_{\alpha}: F_{\alpha} \times{ }_{H_{\alpha}} G \rightarrow G / H_{\alpha}$ are $G \mathscr{F}$ quasifibrations and that the face maps on the simplicial space level are $G \mathscr{F}$ maps.

(ii) is similar to (i), since any map between aspherical spaces is a weak equivalence, and there is a trivial $G$-action on $q^{-1}(x)$ for each $x \in B(\Lambda, \mathcal{Q}, \mathcal{Y}$ ) (since $G$ acts on last coordinates).

(iii) Again similar to (i), using the fact that if $\phi: F \rightarrow F$ is an $H$-homotopy equivalence, then $\phi$ induces a weak equivalence $A_{\alpha}$ W $\rightarrow A_{\alpha}$ W (by $H_{\alpha}$-Whitehead).

In order to ensure that the points in $I(\alpha)$ are nondegenerate, we grow a whisker on each $G \mathscr{F}\left(p_{\alpha}, p_{\alpha}\right)$ at the identity, moving the basepoint to its end, $1 \in[0,1]$, and obtain a new (strict) monoid $\mathbb{Q}^{\prime}$ which acts naturally on any graphs over $\mathbb{Q}$ (see for example [Ma4, A.8]; strictness of $\mathbb{Q}^{\prime}$ follows from strictness of $\mathbb{Q}$ ).

The following fact about the classifying spaces will be needed later:

LEMMA 2.2.4. If $X$ is a simplicial $G$-space with each $X_{n}$ in $G W$ and each $s X_{n} \rightarrow X_{n+1} a G$-cofibration where $s X_{n}$ is the space of degenerate elements, then $|X|$ is in $G \mathscr{W}$. In particular, if $\mathcal{X}$ and $\mathcal{Q}$ are in $\mathscr{W}$ and $\mathcal{Y}$ is in $G \mathscr{W}$, then $B(\mathcal{X}, \mathcal{Q}, \mathcal{Y})$ is in $G$ ข.

The proof is just an application of invariance of pushouts and colimits of cofibrations and of G-CW approximation. (See, e.g., [Ma5, A.4] for a readily adaptable nonequivariant version.)

LEMMA 2.2.5. Suppose that $F$ is compact, and that $(G \mathscr{F}, F)$ is an equivariant category of fibers such that $F$ is in $H_{\alpha}$ W for each $\alpha \in \Lambda$. Then $Q$ is in $W$.

Proof. The maps $\pi_{\alpha \beta}: G \mathscr{F}\left(p_{\alpha}, p_{\beta}\right) \rightarrow \tilde{G} \mathscr{U}\left(p_{\alpha}, p_{\beta}\right)$ are fibrations, and $\tilde{G} \mathscr{U}$ is a union of components of $G \mathcal{U}\left(p_{\alpha}, p_{\beta}\right) \cong N_{H_{\alpha}}\left(H_{\beta}\right)$, a closed manifold, and hence a $\mathrm{CW}$ complex. The result is thus a consequence of [St1] (or [Wa3]).

Corollary 2.2.6. With $(G \mathscr{F}, F)$ as above, the spaces $B(\Lambda, \mathcal{Q}, \mathcal{\theta})$ and $B(\Lambda, \mathcal{Q}, \mathscr{F})$ are in $G$ W.

Corollary 2.2.7. The map $\Gamma p: \Gamma B(\Lambda, \mathcal{Q}, \mathscr{F}) \rightarrow B(\Lambda, \mathcal{Q}, \mathcal{\Theta})$ is a $(G \mathscr{F}, F)$ fibration.

2.3. The classification of $G \mathscr{F} \mathscr{Q}$ - and $G \mathscr{F} \mathscr{W}$-fibrations. Now that we have established the appropriate machinery, we may employ an elaboration of [Ma2, \$9] to obtain a classification theorem. First, some preliminary lemmas.

LEMMA 2.3.1. Let $\mathcal{Q}, \mathcal{X}$ and $\mathbb{Q}$ be as defined in 2.2.2. Then the natural maps

$$
\varepsilon_{\alpha}: B\left(\mathfrak{X}, \mathcal{Q}, \mathbb{Q}_{\alpha}\right) \rightarrow \mathfrak{X}_{\alpha}=T^{-1}(\alpha)
$$

are deformation retractions for each $\alpha \in \Lambda$ (where $T: \mathcal{X} \rightarrow \Lambda$ is the target map).

Proof. This is a consequence of [Ma4, \$§9.8, 9.9 and 11.10] in which a contraction is constructed on the simplicial space level. The maps 


$$
\begin{aligned}
\tau_{\alpha}: \chi_{\alpha} & \rightarrow B\left(\chi, \mathcal{Q}, \mathbb{Q}_{\alpha}\right), \\
x & \mapsto\left(x[] 1_{\alpha},(1)\right)
\end{aligned}
$$

are homotopy inverses.

LEMMA 2.3.2. Let $(G \mathscr{F}, F)$ be the equivariant category of fibers $(G \mathscr{F} \mathscr{Q}, F)$ or $(G \mathcal{F} \mathscr{W}, F)$ with $F \in H_{\alpha}$ $W$ for each action $\alpha \in \Lambda$. If $p: E \rightarrow B$ is $a G \mathcal{F}$ fibration, then $\bar{\varepsilon}$ in the following diagram is a weak $G$-equivalence:

$$
\begin{array}{ccc}
E & \stackrel{\varepsilon}{\leftarrow} & B(\mathcal{P}(E), \mathcal{Q}, \mathscr{F}) \\
p \downarrow & & p \downarrow \\
B & \stackrel{\bar{\varepsilon}}{\leftarrow} & B(\mathscr{P}(E), \mathcal{Q}, \mathcal{O})
\end{array}
$$

where $\mathcal{P}(E), \mathcal{Q}, \mathcal{O}$ and $\mathcal{F}$ are given by Example 2.2.2.

Proof. Applying the functors $P()_{\alpha}$, we obtain:

$$
\begin{array}{ccc}
P(E)_{\alpha} & \stackrel{\varepsilon_{\alpha}}{\leftarrow} & B\left(\mathcal{P}(E), \mathcal{Q}, \mathbb{Q}_{\alpha}\right) \\
P(P)_{\alpha} \downarrow & & \downarrow \\
P(B)_{\alpha} & \stackrel{\varepsilon_{\alpha}}{\leftarrow} & B\left(\mathcal{P}(E), \mathcal{Q}, \tilde{\mathbb{Q}}_{\alpha}\right)
\end{array}
$$

for each $\alpha \in \Lambda$, where the $\varepsilon_{\alpha}$ are equivalences. Thus, by Lemma 2.3.1 and the five-lemma, we conclude that each $\bar{\varepsilon}_{\alpha}$ is a weak equivalence.

We must now deduce that

$$
\bar{\varepsilon}^{H}: B(\mathscr{P}(E), \mathcal{Q}, \mathcal{O})^{H} \rightarrow B^{H}
$$

is a weak equivalence for each closed subgroup $H$. This is seen as follows:

First, $P(B)_{\alpha}$ is a union of components of $B^{H_{\alpha}}$ (because $p$ is a $(G \mathscr{F}, F)$ fibration). Now $B\left(\mathscr{P}(E), \mathcal{Q}, \tilde{\mathbb{Q}}_{\alpha}\right)$ consists of points $\left(\left(\theta_{0}, \bar{\theta}_{0}\right),\left[\left(\theta_{1}, \bar{\theta}_{1}\right), \ldots,\left(\theta_{n}, \bar{\theta}_{n}\right)\right], \theta_{n+1}, s\right)$ where the $\theta_{i}$ and $\bar{\theta}_{i}$ fit into a diagram as follows:

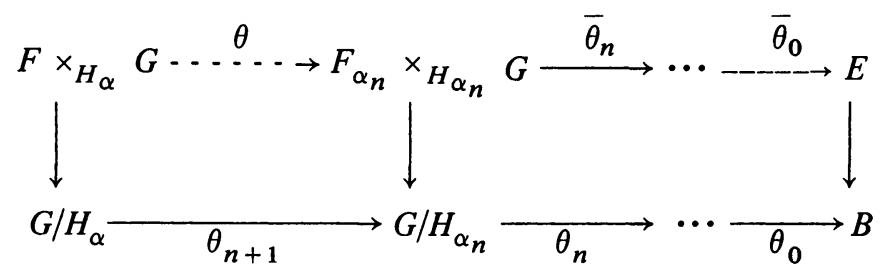

and $s \in \Delta^{n}$. Points of $B(\mathscr{P}(E), \mathcal{Q}, \mathcal{\theta})^{H_{\alpha}}$, regarded as $G$-maps $G / H_{\alpha} \rightarrow$ $B(\mathscr{P}(E), \mathcal{Q}, \mathcal{O})$, may be represented in exactly the same way, except that a covering $G \mathscr{F}$ map $\theta$ need not exist.

We now assert that $B\left(\mathscr{P}(E), Q, \tilde{\mathbb{Q}}_{\alpha}\right)$ is a union of components of $B(\mathscr{P}(E), \mathcal{Q}, \mathcal{O})^{H_{\alpha}}$. Indeed, if

$$
\lambda: I \rightarrow B(\mathscr{P}(E), \mathcal{Q}, \mathcal{O})^{H_{\alpha}}
$$

is a path starting in $B\left(\mathscr{P}(E), \mathbb{Q}, \tilde{\mathbb{Q}}_{\alpha}\right)$, then $\lambda$ gives a homotopy

$$
\bar{\phi}_{1} \sim \bar{\phi}_{2}: G / H_{\alpha} \times I \rightarrow B \quad(\text { via } \overline{\mathcal{E}})
$$

such that $\bar{\phi}_{1}$ defines a diagram as above; 


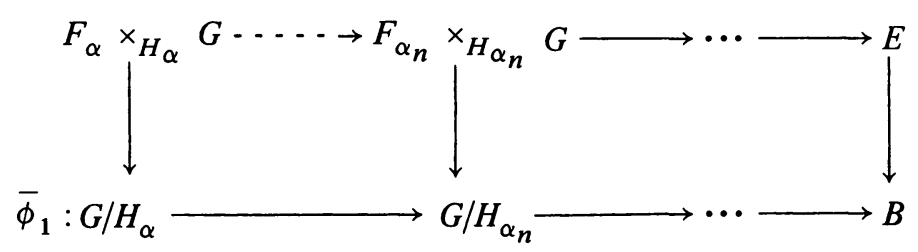

while $\bar{\phi}_{2}$ defines a similar diagram, except that the dotted arrow is not given to exist.

The $G \mathscr{F}$-CHP gives a map $\gamma$ in the following diagram:

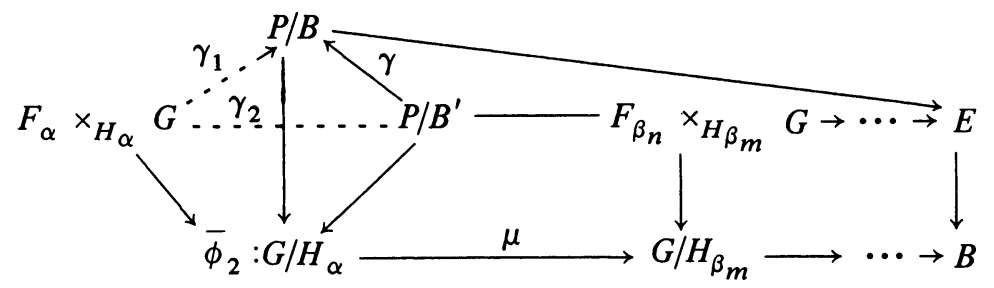

in which $P / B$ and $P / B^{\prime}$ are the evident pullbacks, $\gamma$ and $\gamma_{1}$ are the universal $G$-maps, and the sequence on the bottom is defined by $\bar{\phi}_{2} \cdot \gamma_{2}$ exists by the equivariant Whitehead Theorem applied to the fiberwise weak equivalence $\gamma$. Hence, by the diagram, $\lambda(1) \in B\left(\mathcal{P}(E), \mathbb{Q}, \tilde{\mathbb{E}}_{\alpha}\right)$, since the map $\mu$ is covered by a $G$ map.

Next, we show that $\bar{\varepsilon}^{H_{\alpha}}$ is bijective on components. The result will follow, since $\bar{\varepsilon}_{\alpha}$ is a weak equivalence.

First, $\bar{\varepsilon}^{H_{\alpha}}$ is surjective on components because it is clearly a surjection. Injectivity is more tricky; let $\lambda: I \rightarrow B^{H_{\alpha}}$ be a path from a point $p$ in the image of $B\left(\mathscr{P}(E), \mathcal{Q}, \tilde{\mathbb{Q}}_{\alpha}\right)$ to a point $q$ in the image of $B\left(\mathcal{P}(E), \mathbb{Q}, \tilde{\mathbb{Q}}_{\beta}\right)$, where $H_{\beta}=H_{\alpha}=$ $H$. Since $\bar{\varepsilon}_{\alpha}$ is a weak equivalence for each $\alpha \in \Lambda$, we may assume that $p$ and $q$ are of the form $\left(f_{\alpha}[] 1_{\alpha},(1)\right)$ and $\left(f_{\beta}[] 1_{\beta},(1)\right)$ respectively, where $f_{\alpha}: F_{\alpha} \times{ }_{H_{\alpha}} G \rightarrow E$ and $f_{\beta}: F_{\beta} \times{ }_{H_{\beta}} G \rightarrow E$ arise from $G \mathscr{F}$ maps. Now the $G \mathscr{F}$ lifting property applied to $\lambda$ and $f_{\alpha}$ gives an $H$-equivalence $\psi: F_{\alpha} \rightarrow F_{\beta}$. Let $\bar{f}_{\alpha}=f_{\beta} \circ \psi \times{ }_{H}$. Then $\bar{f}_{\alpha}$ is $G$-homotopic to $f_{\alpha}$ over the map $\lambda(1): G / H_{\alpha} \rightarrow B$, because of the way $\psi$ was constructed. This gives a path from $p$ to $\left(\bar{f}_{\alpha}[] 1_{\alpha},(1)\right)$, which may then be joined to $q$ by the path $t \mapsto\left(f_{\beta}\left[\psi \times_{H} 1\right] 1_{\alpha},(t, 1-t)\right)$, as required.

LEMMA 2.3.3. With $F$ and $G \mathscr{F}$ as in 3.3.2, the natural map

$$
\eta: B(\Lambda ; \mathbb{Q}, \mathscr{F}) \rightarrow \Gamma B(\Lambda, \mathbb{Q}, \mathscr{F})
$$

induces weak equivalences

$$
P(\eta)_{\alpha}: P(B(\Lambda, \mathcal{Q}, \mathscr{F}))_{\alpha} \rightarrow P(\Gamma B(\Lambda, \mathcal{Q}, \mathscr{F}))_{\alpha}
$$

for each $\alpha \in \Lambda$.

Proof. There are natural homeomorphisms making the following diagram commute for each $\alpha \in \Lambda$ :

$$
\begin{array}{ccc}
P(B(\Lambda, \mathcal{Q}, \mathscr{F}))_{\alpha} & \cong & B\left(\Lambda, \mathcal{Q}, \mathbb{Q}_{\alpha}\right) \\
P(q)_{\alpha} \downarrow & \downarrow q_{\alpha} \\
P(B(\Lambda, \mathcal{Q}, \mathcal{O}))_{\alpha} \cong & \simeq B\left(\Lambda, \mathcal{Q}, \tilde{\mathbb{Q}}_{\alpha}\right)
\end{array}
$$


Thus $P(q)_{\alpha}$ is a quasifibering. Further, the map $P(\eta)_{\alpha}$ in the hypothesis restricts to a weak equivalence on each fiber (by the $\Gamma$-completeness of $(G \mathscr{F}, F)$ ). The conclusion follows by an application of the five-lemma to the horizontal arrow in the diagram:

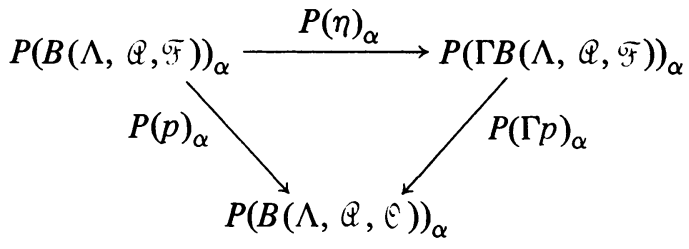

where the two (apparently distinct) interpretations of $P(B(\Lambda, \mathcal{Q}, \vartheta))_{\alpha}$; one from $p$ and the other from $\Gamma p$, are the same.

Corollary 2.3.4. $P(\Gamma B(\Lambda, \mathcal{Q}, \mathscr{F}))_{\alpha}$ is aspherical and nonempty for each $\alpha \in \Lambda$.

Proof. A consequence of 3.3.1, 3.3.3 and the homeomorphisms $P(B(\Lambda, \mathcal{Q}, \mathscr{F}))_{\alpha}$ $\simeq B\left(\Lambda, \mathbb{Q}, \mathbb{Q}_{\alpha}\right)$.

Definition 2.3.5. If $(G \mathscr{F}, F)$ is an equivariant category of fibers, let $\mathcal{E} G \mathscr{F}(B)$ denote the collection of equivalence classes of $G \mathscr{F}$ fibrations over $B$ under the equivalence relation generated by the $G \mathscr{F}$ maps over $B$.

Observe that $\mathscr{E} G \mathscr{F} \mathscr{W}(B)$ is the collection of equivalence classes of $G \mathscr{F} \mathscr{W}$ fiberings over $B$ under the relation of fiberwise homotopy equivalence provided $B$ is $G$-numerable.

We now prove the classification theorem.

TheOREM 2.3.6. Let $(G \mathscr{F}, F)$ denote one of the categories $(G \mathscr{F} \mathscr{Q}, F)$ or $(G \mathscr{F} \mathscr{W}, F)$, with $F \in H_{\alpha}$ W for each $\alpha \in \Lambda$, and $F$ compact for the case $G \mathscr{F}=$ $G \mathcal{F} \mathscr{W}$. Then there is a natural isomorphism

$$
\Phi: \mathcal{E} G \mathcal{F}(B) \cong h G \mathscr{Q}(B, B(\Lambda, \mathcal{Q}, \mathcal{O}))
$$

whenever $B \in G$ W.

Proof. (a) Definition of $\Phi$. Let $p: E \rightarrow B$ be a $G \mathscr{F}$ fibration. By 2.3.2, the map $\bar{\varepsilon}$ : $B(\mathscr{P}(E), \mathcal{Q}, \Theta) \rightarrow B$ is a weak- $G$-equivalence. Let $\gamma: X \rightarrow B(\mathcal{P}(E), \mathcal{Q}, \mathcal{O})$ be a $G-C W$ approximation to $B(\mathcal{P}(E), \mathcal{Q}, \mathcal{O})$ (see [Wa2]). Then, by the $G$-Whitehead Theorem, $\bar{\varepsilon} \circ \gamma$, then $\gamma \circ \alpha=G$ is a right $G$-homotopy inverse to $\bar{\varepsilon}$. Let $\Phi(p)$ be given by the composite $B \underset{g}{\rightarrow} B(\mathcal{P}(E), \mathcal{Q}, \mathcal{O}) \underset{p}{\rightarrow} B(\Lambda, \mathcal{Q}, \mathcal{O})$.

(b) Definition of $\Psi=\Phi^{-1}$. Given a $G$-map $f: B \rightarrow B(\Lambda, \mathcal{Q}, \theta)$, let $\Psi(f)$ denote the $G$ fibration obtained by pulling back $\Gamma p: \Gamma B(\Lambda, \mathcal{Q}, \theta) \rightarrow B(\Lambda, \mathcal{Q}, \theta)$ over $f$.

(c) Proof that $\Psi \Phi=1$. Consider the following commutative diagram of $G \mathscr{F}$ spaces:

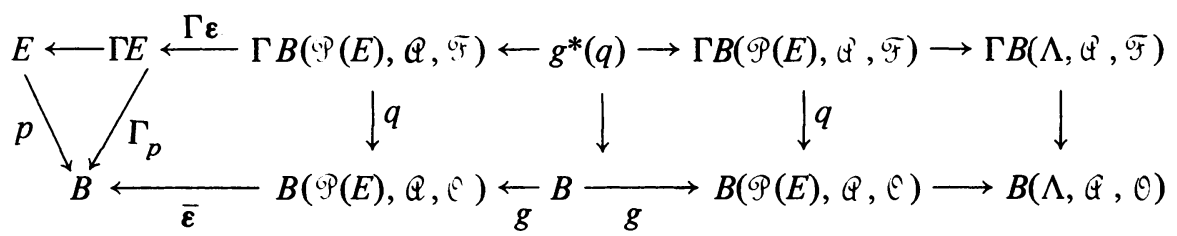


Since $\bar{\varepsilon} \circ g \sim 1_{B}$, the $G \mathscr{F}-C H P$ gives a map $g^{*}(q) \rightarrow \Gamma E$ over $B$ (of $G \mathscr{F}$ fiberings). By the universal property of pullbacks and by the diagram, there is a $G \mathscr{F}$ map $g^{*}(q) \rightarrow \Psi \Phi(p)$ over $B$. This shows that $\Psi \Phi=1$, the case of $G \mathscr{F} \mathscr{W}$ being dealt with by the generalization of Stasheff's work in [Wa3], so that all fibers over points of $B$ are equivariant $C W$ under the appropriate subgroups.

(d) Proof that $\Phi \Psi=1$. Given $f: B \rightarrow B(\Lambda, Q, \mathcal{Q})$, we consider the following diagram:

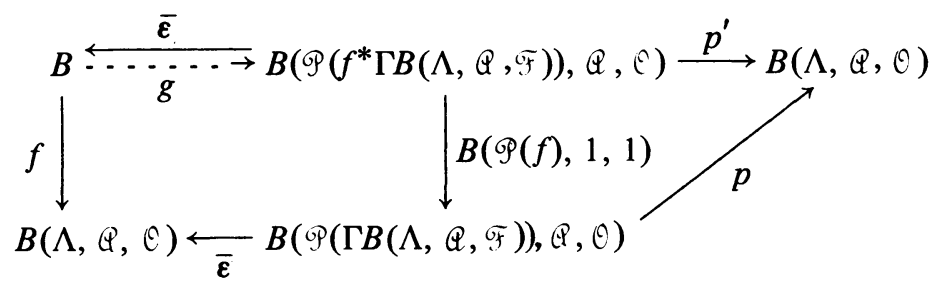

The argument in Lemma 2.3.2 shows that the bottom map $\bar{\varepsilon}$ is a weak- $G$-equivalence. Further, by Proposition 2.2.3(ii) and Corollary 2.3.4 the map $p$ is also a weak- $G$-equivalence.

Thus, by the diagram, $\Phi \Psi$ is an automorphism of $h G \mathcal{Q}(B, B(\Lambda, \mathcal{Q}, \mathcal{O}))$, whence $\Psi$ is a bijection, and hence the inverse of $\Phi$.

2.4. Comments on the universal base spaces. In [La1], a relation is displaced between the fixed point sets of the universal base spaces for equivariant bundles and the classifying spaces for the various representations. Here, we prove a generalization of this result for $(G \mathscr{F}, F)$ fibrations.

THEOREM 2.4.1. Let $(G \mathscr{F}, F)$ be one of the categories of fibers $(G \mathscr{F} \mathscr{Q}, F)$ or $(G \mathscr{F} \mathscr{W}, F)$. Then, if $H \subset G$ is a closed subgroup, $B(\Lambda, \mathcal{Q}, \mathcal{O})^{H}$ is weakly homotopy equivalent to $\amalg_{\alpha \in R_{H}} B A_{\alpha}$, where $R_{H}$ is a set of representatives of homotopy equivalence classes of actions of $H$ on $F$ such that $F \in H$ W (so that $R_{H}=\Lambda_{H} / \sim$, where $\Lambda_{H}=\left\{\alpha \in \Lambda ; H_{\alpha}=H\right\}$ and $\alpha \sim B \Leftrightarrow F_{\alpha}$ and $F_{\beta}$ are $H$-homotopy equivalent).

Proof. It clearly suffices to show that $X=\amalg_{\alpha \in R_{H}} B\left(*, A_{\alpha}, *\right)$ is the classifying space for $(H \mathcal{F}, F)$ fibrations over $H$-trivial base spaces (a comparison between $X$ and $B(\Lambda, Q, \mathcal{O})^{H}$ being given by the obvious inclusion). But this is a straightforward generalization of the nonequivariant classifying theorem using the categories of fibers $\left(\mathscr{F}_{\alpha}, F\right)$ associated with the actions considered, proved in [Ma2]. (Alternatively, we repeat the equivariant classification theorem using the equivariant category of fibers whose distinguished objects are $\left\{F_{\alpha}\right\}_{\alpha \in R_{H}}$ regarded as $H$-maps $p_{\alpha}: F_{\alpha} \rightarrow H / H=*$.)

This gives us a formulation of classifying spaces for Illman's equivariant singular cohomology theories [II1] in restricted situations. It has become apparent that equivariant cohomology theories indexed by $R O(G)$ play an important role in equivariant stable homotopy, and the construction of an equivariant singular cohomology theory with 0 th representing space $B(\Lambda, \mathcal{Q}, \mathcal{\theta})$ and naturally indexed by $R O(G)$ is work in progress. 


\section{Based fibrations, bundles, and stable fibrations.}

3.1. Based $G$-fibrations. By far the most interesting class of equivariant fiberings is that of spherical fiberings whose fibers are based $G$-spaces (where $G$ acts trivially on the basepoint). Classification of these objects is, in effect, already done for us in $\$ 2$ and [Ma2]; all one need do is carry out the purely formal adaptation of 2.1, 2.2 and 2.3, replacing $\Gamma$ by $\Gamma^{\prime}$ as in [Ma2, §5].

We shall denote the based equivariant categories of fibers corresponding to $G \mathscr{F} \mathscr{U}$ and $G \mathscr{F} \mathscr{W}$ by $G \mathscr{F} \mathcal{T}$ and $G \mathscr{F} \mathscr{V}$ respectively, $\mathcal{T}$ being the category of based spaces in $\mathscr{Q}$, and $\mathfrak{V}$ being the category of based spaces in $\mathscr{W}$.

3.2. Equivariant categories of bundle fibers. The purpose of this section is to show how equivariant fiber bundles (as discussed in [La1, §1] for example) are, under suitable conditions, $G \mathscr{F}$ fibrations for certain categories of fibers $G \mathscr{F}$.

Definition 3.2.1. A $(G, A)$ bundle is a $G$-map $p: E \rightarrow B$ which is a bundle with structure group $A$ and with fiber $F$ (in the sense of Steenrod) such that $G$ acts on $E$ through $A$ bundle maps. A locally trivially $(G, A)$ bundle is a $(G, A)$ bundle $p$ : $E \rightarrow B$ such that $B$ has a cover by open tubes $G V_{\alpha}$ such that $p: p^{-1}\left(G V_{\alpha}\right) \rightarrow G V_{\alpha}$ is $(G, A)$ equivalent to

$$
G \times{ }_{H_{\alpha}}\left(V_{\alpha} \times F\right) \rightarrow G \times_{H_{\alpha}} V_{\alpha} \simeq G V_{\alpha}
$$

for some action of $H_{\alpha}$ through $A$, and some topological space $F$.

DefinITION 3.2.2. Let $F$ be a space in $\mathcal{U}$, and let $A$ be a topological group which acts effectively on $F$ (from the right). Define an equivariant category of fibers $(G A \mathscr{B}, F)$ as follows:

The objects of $G A \mathscr{B}$ are pairs $(p, \Phi)$, where $p: p \rightarrow Q$ is a $G$-map onto an orbit with $A$ acting on each fiber, and $\Phi \in G \mathscr{F} \mathscr{U}\left(p_{\alpha}, p\right)$ is an $A$-homeomorphism on fibers for some action $\alpha \in G \mathscr{F} \mathscr{U}$ such that $\alpha: H_{\alpha} \rightarrow$ Aut $F_{\alpha}$ factors as a representation $\bar{\alpha}: H_{\alpha} \rightarrow A$. (Recall $p_{\alpha}: F \times{ }_{H_{\alpha}} G \rightarrow G / H_{\alpha}$ )

The morphisms of $G A \mathscr{B}$ are those morphisms in $G \mathcal{F} \mathscr{Q}$ of the form $\Phi^{\prime} \hat{a} \Phi^{-1}$ : $(p, \Phi) \rightarrow\left(q, \Phi^{\prime}\right)$, where $\hat{a}: p_{\alpha} \rightarrow p_{\beta}$ is a morphism in $G \mathscr{F} \mathscr{Q}\left(p_{\alpha}, p_{\beta}\right)$ which is given on $p_{\alpha}^{-1}(e)$ by multiplication by $f \rightarrow(f a, g)$, for some element $(a, g) \in A \times{ }_{H_{\beta}} G$.

By a $G$-numerable space, we shall mean a $G$-space $B$ with a $G$-partition of unity subordinate to a cover of $B$ by open tubes.

THEOREM 3.2.3. Suppose that $B$ has a cover by open tubes as well as a G-partition of unity which enumerates this cover. If $p: E \rightarrow B$ is $a(G, A)$ locally trivial bundle with fiber $F$, then $p$ is a $(G A \mathscr{B}, F)$ fibration.

Proof. By [La1, §1.2], all $(G, A)$ locally trivial bundles satisfy the $G$-CHP. Alternatively, we appeal to the following:

Lemma 3.2.4. Let $p: E \rightarrow B$ be $a(G \mathscr{F}, F)$ space, and assume that $B$ admits $a$ numerable $G$-cover $\mathcal{C}$ such that $p \mid p^{-1}(U)$ is a $(G \mathcal{F}, F)$ fibration for each $U \in \mathcal{C}$. Then $p$ is a $(G \mathcal{F}, F)$ fibration.

The proof is an easy generalization of [Ma2, §3.8].

We now wish to assert that $G A \mathscr{B}$ fibrations may be classified as in $\$ 2$. The following proposition will demonstrate that gammafication is not necessary: 
Proposition 3.2.5. Let $(G \mathscr{F}, F)=(G A \Re, F)$. Then, with the notations of $\S 2$, the map

$$
p: B(\mathscr{X}, \mathcal{Q}, \mathscr{F}) \rightarrow B(\mathcal{X}, \mathcal{Q}, \mathcal{O})
$$

is a $(G, A)$ locally trivial bundle, hence $(G A \mathfrak{B}, F)$ fibration, whenever $\mathscr{X}$ is numerable.

Proof. By Theorem 3.2.3, it suffices to show that $p$ is locally $(G, A)$ trivial over some numerable $G$-cover $\mathcal{C}$ of $B(\mathcal{X}, \mathcal{Q}, \mathcal{\theta})$.

Generalizing [Ma2, §8.2], we define a partition of unity $\left\{\pi_{n}\right\}$ as follows:

Let $B=B(\mathscr{X}, \mathcal{Q}, \mathcal{O})$. Then a consideration of simplicial coordinates shows that $\left(B, F_{n} B\right)$ admits a $G$-representation $\left(h_{n}, u_{n}\right)$ as a $G$-NDR pair. For $n>0$, define $G$-maps $\rho_{n}: B \rightarrow I$ by

$$
\begin{aligned}
& \rho_{0}(x)=1-u_{0}(x), \text { and } \\
& \rho_{n}(x)=\left(1-u_{n}(x)\right) u_{n-1}\left(h_{n}(x, 1)\right) \text { if } n>0 .
\end{aligned}
$$

Then define

$$
\pi_{n}(x)=\max \left(0, \rho_{n}(x)-n \sum_{i=0}^{n-1} \rho_{i}(x)\right) .
$$

$\pi_{n}$ is then seen to be a $G$-partition of unity for $B$.

If $W_{n}=\pi_{n}^{-1}(0,1], p \mid E_{W_{n}}$ is seen to be $(G, A)$ bundle by the argument in [Ma2, \$8.2].

In a similar way, we obtain the analogous result for the associated principal fiberings.

This shows that it will not be necessary to use gammafications to obtain fibrations (because the universal "quasifibrations" will, in fact, already be bundles).

Proposition 3.2.6. With the above notations, the maps

$$
p_{\alpha}: B\left(\mathcal{X}, \mathbb{Q}, \mathbb{Q}_{\alpha}\right) \rightarrow B\left(\mathcal{X}, \mathbb{Q}, \mathbb{Q}_{\alpha}\right)
$$

are locally trivial $A_{\alpha}$ bundles for each $\alpha \in \Lambda$, where $A_{\alpha} \subset A$ is the space of all $H_{\alpha}$-equivariant maps $F_{\alpha} \rightarrow F_{\alpha}$.

Proof. This is completely analogous to 3.2 .5 and uses the fact that the map

$$
\hat{a}_{0}: H_{\alpha} \mathcal{U}\left(F_{\alpha}, F_{\alpha}\right) \rightarrow H_{\alpha} \mathscr{U}\left(F_{\alpha}, X\right)
$$

is a homeomorphism for any $H_{\alpha}$ space $X$ and any $H_{\alpha}$ homeomorphism $\hat{a}_{0}: F_{\alpha} \rightarrow X$. (This replaces the Whitehead result used in the cases studied in \$2.)

The classification theorem 2.3.6 then goes through automatically, where we omit $\Gamma$ throughout, and we obtain:

TheOREM 3.2.7. ( $G A \mathscr{B}, F)$ fibrations are classified by $B(\Lambda, \mathcal{Q}, \mathcal{O})$, where $\mathcal{Q}, \mathcal{O}$ and $\Lambda$ are the relevant graphs for the category $G A \mathscr{B}$ (described in \$2).

This is not quite a classification theorem for bundles, but closely related to one. Since the morphisms $\hat{a}: p_{\alpha} \rightarrow p_{\beta}$ in $G A \mathscr{B}$ are formed from the $A$-action on $F$, we may retopologize $G A \mathscr{B}\left(p_{\alpha}, p_{\beta}\right)$ as a subspace of $A \times{ }_{H_{\beta}} G$ and thereby obtain newly topologized spaces $\tilde{B}(\Lambda, \mathcal{Q}, \mathcal{O}) ; 3.2 .5$ and 3.2.6 remain valid. 
THeOREM 3.2.8. Let $A$ be a topological group which acts effectively on $F$ (from the right), and let $G \mathscr{B} \mathscr{F}(B)$ denote the set of $G$-equivalence classes of numerable $(G, A)$ bundles with fiber $F$ over $B$. Then, for $B \in G \mathscr{W}, G \mathscr{B} \mathscr{F}(B)$ is naturally isomorphic to $h G \mathcal{Q}(B, \tilde{B}(\Lambda, \mathcal{Q}, \mathcal{\theta}))$.

Proof. This is formally identical to 3.2.7 in view of 3.2.5 and 3.2.6, where we topologize the function spaces using the topology of $A$.

COROLLARY 3.2.9. Suppose that the natural map from the appropriate subspace of $A \times{ }_{H_{\beta}} G$ to $G A \mathscr{B}\left(p_{\alpha}, p_{\beta}\right)$ (with the compact-open-topology) is a weak equivalence for each $\alpha$ and $\beta$. Then the natural transformation

$$
\rho: G \mathscr{B} \mathscr{F}(B) \rightarrow \mathcal{E} G A \mathscr{B}(B)
$$

is a bijection.

The proof simply amounts to the observation that $\rho$ is represented by the evident map

$$
\tilde{B}(\Lambda, \mathcal{Q}, \mathcal{\theta}) \rightarrow B(\Lambda, \mathcal{Q}, \mathcal{\theta})
$$

which is a weak equivalence under the given hypotheses (which always hold in practice); compare [Ma2, \$9.11].

3.3. Stable G-fibrations.

3.3.1. NotATIONS AND BASIC NOTIONS. Fix a $G$ action on $\mathbf{R}^{\infty}$ such that:

(i) each finite dimensional real orthogonal representation of $G$ appears infinitely of ten in $\mathbf{R}^{\infty}$ (up to equivalence);

(ii) $\mathbf{R}^{\infty}$ is the colimit of its finite dimensional invariant subspaces.

Denote $\mathbf{R}^{\infty}$ with this action by $G \mathbf{R}^{\infty}$.

The letters $V, W$ and $U$ will be used to denote finite dimensional invariant subspaces of $\mathbf{R}^{\infty}$, and $S^{V}$ will denote the one-point compactification of $V$, based at infinity.

Let $\Sigma^{V} X=X \wedge S^{V}$ for based $G$-spaces $X \in G \tau$, and let $\Omega^{V} X$ be the space of based maps $S^{V} \rightarrow X$. $G$ acts on both $\Sigma^{V} X$ and $\Omega^{V} X$ in the evident ${ }^{*}$ ways. Observe that the adjunctions $\varepsilon_{v}: 1 \rightarrow \Omega^{V} \Sigma^{V}$ and $\xi_{v}: \Sigma^{V} \Omega^{V} \rightarrow 1$ restrict to adjunctions of endofunctors on $G \tau$.

The following proposition motivates the choice of an ambient $G$-space $G \mathbf{R}^{\infty}$ as opposed to use of the category of finite dimensional orthogonal $G$ modules and linear embeddings:

Proposition and Definition 3.3.2. Let $G \mathcal{G}_{*}$ denote the category whose objects are the finite dimensional invariant subspaces of $G \mathbf{R}^{\infty}$ and whose morphisms are inclusions of such spaces. A $G \mathcal{G}_{*}$ functor $T$ is a continuous functor $T: G \mathcal{G}_{*} \rightarrow G \tau$ such that $T(\iota)$ is a closed inclusion for every morphism $\iota$ in $G \mathcal{G}_{*}$. If $X$ is a compact $G$-space $G \tau$, then the natural maps

$$
\nu: \operatorname{colim} G \tau(X, T(V)) \rightarrow G \tau(X, \operatorname{colim} T(V))
$$

and

$$
h \nu: \operatorname{colim} h G \tau(X, T(V)) \rightarrow h G \tau(X, \operatorname{colim} T(V))
$$

are isomorphisms. 
Proposition 3.3.2 follows from the existence of a cofinal sequence of finite dimensional $G$-subspaces of $\mathbf{R}^{\infty}$.

3.3.3. EXAMPLes of $G \mathcal{G}_{*}$ Functors. (i) Let $T(V)=\Omega^{V} \Sigma^{V} X$ for some $X \in G \tau$, with the action of $T$ on morphisms in $G \mathcal{G}_{*}$ the usual map $T(\iota): \Omega^{V} \Sigma^{V} X \rightarrow \Omega^{W} \Sigma^{W} X$ (for $\iota: V \rightarrow W$ the inclusion of a subspace $V$ ).

(ii) Let $F \in G$ Q be a space (given a preferred $G$-action; possibly trivial), and let $T(V)$ denote the classifying space for the category of fibers $\left(G \mathscr{F} \mathscr{V}, \Sigma^{V} F^{+}\right)$, with the $T$ action on inclusions $\iota: V \rightarrow W$ being given by suspension with the complement of $V$ in $W$. (The reason for a preferred action on $F$ will become evident shortly.)

DEFINITION 3.3.4. A spherical $G$-fibering is a $\left(G \mathscr{F} \mathcal{V}, S^{V}\right)$ fibration for some $V \subset G \mathbf{R}^{\infty}$. Two spherical $G$-fiberings over $X$ are stably $G$-equivalent, $\xi \sim \xi^{\prime}$, if $\Sigma_{\mathscr{F}}^{W} \xi$ is fiberwise $G$-homotopy equivalent to $\Sigma_{\mathscr{F}}^{W^{\prime}} \xi^{\prime}$ for some $W, W^{\prime} \subset G \mathbf{R}^{\infty}$, where $\Sigma_{\mathscr{F}}^{W}$ denotes fiberwise suspension by $S^{W}$.

When the base space $X$ is based by adding a disjoint basepoint + , a preferred $G$-action on $S^{V}$ is selected by any spherical $G$-fibering over $X^{+}$, and relativity (in $R O(G)$ ) is preserved by stable $G$-equivalence. (That is, fiberwise suspension of a fibraton over $*^{+}$gives the same element of $R O(G)$.) Formally, we have the following:

Let $\operatorname{Sph}_{G}(X)$ denote the set of equivalence classes of stable $G$-fibrations over $X^{+}$.

THEOREM 3.3.5. Let $F(V)$ be the classifying space for $\left(G \mathscr{F} \mathfrak{V}, S^{V}\right)$ fibrations (as in Example 3.3.3(ii)) based at $\left(*[] M_{e_{V}},(0)\right)$, where $e_{V}$ is the point (identity coset) in $G / H_{\alpha}$ for $H_{\alpha}=G$ with action $\alpha$ that of $G$ on $V$. Then the natural map

$$
\Phi: h G \mathcal{T}\left(X^{+}, \operatorname{colim} T(V)\right) \rightarrow \operatorname{Sph}_{G}(X)
$$

is an isomorphism.

The proof is a formal consequence of the definitions.

REMARKS 3.3.6. (i) This is the analogue of reduced equivariant $K$-theory, $\tilde{K}_{G}(X)$, where the analogue for unreduced equivariant $K$-theory, $K_{G}(X)$ is defined to be

$$
h G \tau\left(X^{+}, \operatorname{colim} T(V) \times \mathbf{Z}\right) .
$$

(ii) The definitions work more generally, and there is no need to restrict attention to the particular categories of fibers $\left(G \mathscr{F} \mathscr{V}, S^{V}\right)$. Since all the actions in the image of the equivariant $J$-homomorphism are orthogonal, it is useful to consider $\left(G \mathcal{L}, S^{V}\right)$, the equivariant category of fibers obtained from $\left(G \mathscr{F} \mathscr{V}, S^{V}\right)$ by restricting attention to orthogonal linear $G$-actions. We denote the stable classifying space for these categories by $B_{G} F$. The analogous bundle category is $(G O(V) \mathscr{B}, V)$.

Precisely analogous arguments then give us stable classifying spaces in these cases; the last two are $B_{G}$ Top and $B_{G} O$, and we obtain $B_{G} U$ similarly.

In order to specify the more interesting categories of fibers pertinent to stable $G$-theory, we establish some notation.

3.3.7. Notations AND EXAMPles. (i) As in 3.3.6, let $B_{G} F$ denote the classifying 
space for the stable spherical $G$-fibrations derived from the categories $\left(G \mathcal{E} \mathcal{V}, S^{V}\right)$; namely

$$
B_{G} F=\operatorname{colim} B_{G} F(V)
$$

(where $B_{G} F(V)$ is the classifying space for $\left(G \mathscr{L} \mathfrak{V}, S^{V}\right)$ fibrations).

(ii) Let $Q X$ denote the $G$-space colim $\Omega^{V} \Sigma^{V} X$ for $X \in G \tau$, and let $F^{G}$ denote the subspace of $\left(Q S^{0}\right)^{G}$ consisting of the $G$-homotopy equivalences. Since $F^{G}$ is a grouplike topological monoid, we have $\Omega B\left(F^{G}\right) \simeq F^{G}$, and by the usual arguments, we obtain $B\left(F^{G}\right) \simeq\left(B_{G} F\right)^{G}$. In fact, as a result of [Br1, §0], we see that $\left(B_{G} F\right)^{H}=$ $B\left(F^{H}\right)$, since $G \mathbf{R}^{\infty}$, regarded as a space acted on by $H$, satisfies the original hypotheses for the ambient space for $H$.

It follows as in $\$ 2.4$ that the equivariant classifying spaces $B_{G} F$ have the property that their fixed point sets $\left(B_{G} F\right)^{H}$ are standard classifying spaces for equivariant stable spherical fibrations over a trivial $G$-space.

3.4. Some remarks on equivariant $K$-theory. The arguments in 3.3 establish the following:

Proposition 3.4.1. $B_{G} O$ and $B_{G} U$ are the classifying spaces for (reduced) equivariant $K$-theory (as one would hope).

In 3.5, we shall construct alternate models for $B_{G} O, B_{G} U$ and $B_{G} F$ which have important internal structure.

For $V<G \mathbf{R}^{\infty}$ a $G$-subspace, let $O(V)$ denote the space of orthogonal $G$-maps $V \rightarrow V$, and let $O^{G}=$ colim $O(V)$. Then standard classifying-space arguments show that the natural map $\iota: B\left(O^{G}\right) \rightarrow\left(B_{G} O\right)^{G}$ is a $G$-equivalence. Similarly, by 3.3.7(ii), $\iota: B\left(F^{G}\right) \rightarrow\left(B_{G} F\right)^{G}$ is also a $G$-equivalence.

Recall the following result of tom Dieck [Di1]: Let $A(G)$ denote the Burnside ring of $G$, and let $A(G)^{*}$ be the group of units in $A(G)$. Then there is an isomorphism

$$
d: \pi_{0}\left(F^{G}\right) \simeq A(G)^{*}
$$

By [Ma2, §8.7], applied to the evident (nonequivariant) category of fibers whose classifying space is $B\left(F^{G}\right)$, we then have the following:

Proposition 3.4.2. (i) There is a weak equivalence

$$
F^{G} \rightarrow \Omega B\left(F^{G}\right) .
$$

(ii) $\pi_{1} B\left(F^{G}\right) \simeq A(G)^{*}$.

Now the results in [Ma4] imply that $B\left(F^{G}\right)$ is the zeroth space of a spectrum, and thus gives rise to a cohomology theory. Similarly, all the fixed-point sets $\left(B_{G} F\right)^{H}$ may be demonstrated to be zeroth spaces of spectra; this makes plausible the assertion that $B_{G} F$ is an equivariant infinite loop space, and this is proved in [MHW].

3.5. Alternate models for $B_{G} O,\left(B_{G} U\right)$, and $B_{G} F$. Here, we construct models for the above classifying spaces which will be used in a subsequent paper.

Recall the notions of a graph, of an algebra over a graph and of equivariant 
category of fibers from $\S 2$. If $n$ is a natural number, define a category $\left(\mathscr{F}_{n}, S^{n}\right)$ of fibers as follows:

The distinguished objects of $\mathscr{F}_{n}$ are the maps

$$
p_{\alpha}: G S^{V_{\alpha}} \rightarrow G\left\{V_{\alpha}\right\}
$$

where $V_{\alpha}$ is an $n$-dimensional subspace of $G \mathbf{R}^{\infty}$ (not necessarily invariant), $S^{V_{\alpha}}$ is the one-point compactificaton of $V_{\alpha}$, and $\left\{V_{\alpha}\right\}$ is regarded as an element of the $n$-dimensional Grassmannian manifold. $G\left\{V_{\alpha}\right\}$ is the orbit of $\left\{V_{\alpha}\right\}$, and $G S^{V_{\alpha}}$ is the "orbit" of $S^{V_{\alpha}}$ (or the twisted product $S^{V_{\alpha}} \times{ }_{H_{\alpha}} G$, where $H_{\alpha}$ is the isotropy subgroup of $\left.\left\{V_{\alpha}\right\}\right)$.

Note that $G\left\{V_{\alpha}\right\}$ has the form $G / H_{\alpha}$ (for $H_{\alpha}=G_{\left(V_{\alpha}\right)}$ ) and that $F_{\alpha}$ is equivalent to $q_{\alpha}: G \times{ }_{H_{\alpha}} S^{V_{\alpha}} \rightarrow G / H_{\alpha}$. The category of fibers $\mathcal{F}$ is then defined as in 1.1.2(a). Let $\Lambda_{n}, \mathcal{Q}_{n}$ and $\mathcal{O}_{n}$ be the associated graph and algebras (as in 2.2.2). We then have:

LEMMA 3.5.1. $B\left(\Lambda_{n}, \mathbb{Q}_{n}, \Theta_{n}\right)$ is a classifying space for $n$-dimensional spherical fibrations. Here, $\Lambda_{n}, \mathbb{Q}_{n}$ and $\mathcal{O}_{n}$ are topologized as follows: $\Lambda_{n}$ is topologized as a subspace of the n-dimensional Grassmannian manifold of $G \mathbf{R}^{\infty}$, and $\mathbb{Q}_{n}$ and $\hat{\theta}_{n}$ are given the weakest topology such that the natural maps $\mathbb{Q}_{n} \rightarrow \Lambda_{n}^{2}$ and $\mathcal{\theta}_{n} \rightarrow \Lambda_{n}$ are continuous.

Proof. We refer to $\S 2 ; 2.2 .6$ goes through automatically and the evaluation maps of 2.3.1 are still continuous (with the appropriate topology for $P(-)$ ). The proof of Lemma 2.3 .2 adapts easily since any $\boldsymbol{H}$-action on an $\boldsymbol{n}$-dimensional vector space is the restriction of the $G$-action on some (not necessarily invariant) subspace of $G \mathbf{R}^{\infty}$.

Definition 3.5.2. Let $F_{G}(n)$ denote the topological $G$-category whose objects are the $n$-dimensional subspaces of $G \mathbf{R}^{\infty}$ and whose morphisms $S^{V} \rightarrow S^{W}$ are those maps of $f$ such that if $f$ is $H$-invariant, then $f$ is an $H$-homotopy equivalence.

THEOREM 3.5.3. $B F_{G}(n)$ is the classifying space for $n$-dimensional spherical G-fibrations.

Proof. First construct an auxiliary category $\tilde{F}_{G}^{n}$ whose objects are those of $F_{G}^{n}$ but whose morphisms are only those maps $f: S^{V} \rightarrow S^{W}$ such that $G_{\{V\}}<G_{\{W\}}$ and $f$ is $G_{\{V\}}$ equivariant. Now there is a homeomorphism $\Phi: B \tilde{F}_{G}^{n} \simeq B\left(\Lambda_{n}, \mathbb{Q}_{n}, \mathcal{O}_{n}\right)$.

Consider the following diagram:

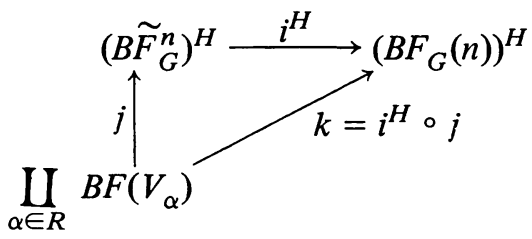

Here, $F\left(V_{\alpha}\right)$ is the grouplike monoid of $H$-equivalence $S^{V_{\alpha}} \rightarrow S^{V_{\alpha}}$ and $R$ is a representative set of $H$-homotopy classes of $n$-dimensional subspaces of $G \mathbf{R}^{\infty}, i^{H}$ is the natural inclusion, as is $j$. But $j$ is an equivalence as a consequence of 2.4.1.

Now $\left(B F_{G}(n)\right)^{H}=B\left(F_{G}(n)\right)^{H}$ is a classifying space for $H$-equivariant fibrations 
over a base space on which $H$ acts trivially (using the category of fibers with distinguished objects $=$ the $\boldsymbol{H}$-invariant compactified $\boldsymbol{n}$-dimensional subspaces of $G \mathbf{R}^{\infty}$ ), and so $k$ is a homotopy equivalence.

It follows that $i^{H}$ is a homotopy equivalence, so that $i$ is a weak- $G$-equivalence, whence a $G$-equivalence by $G$-Whitehead.

We now construct stable versions of the above spaces. Here, care must be taken to obtain well-behaved colimits.

For each invariant subspace $V<G \mathbf{R}^{\infty}$ let $B F_{G}(V)$ be the realization of the category whose objects are the $|V|$ dimensional subspaces of $G \mathbf{R}^{\infty} \oplus V$. Then we may easily pass to colimits to obtain

$$
B F_{G}=\operatorname{colim} B F_{G}(V) \text {. }
$$

Clearly there is a cofinal sequence of invariant subspaces defining the colimit, and $B F_{G}$ classifies stable equivariant spherical fibrations. Similar (and simpler) constructions now give us spaces $B O_{G}$ and $B U_{G}$.

\section{BIBLIOGRAPHY}

[Br1] G. E. Bredon, Introduction to compact transformation groups, Academic Press, New York, 1972.

[Di1] T. tom Dieck, Equivariant stable homotopy theory, Chicago Lecture Notes, 1975.

[II1] S. Illman, Equivariant singular homology and cohomology, Mem. Amer. Math. Soc. no. 156, 1975.

[La1] R. Lashof and M. Rothenberg, G smoothing theory, Proc. Sympos. Pure Math., vol. 32.1, Amer. Math. Soc., Providence, R. I., 1978, pp. 211-266.

[Ma1] J. P. May, Homotopic foundations of algebraic topology, Mimeographed notes, University of Chicago (to appear).

[Ma2] _ Classifying spaces and fibrations, Mem. Amer. Math. Soc. no. 155, 1975.

[Ma3] —_, $E_{\infty}$ spaces group completions and permutative categories, London Math. Soc. Lecture Note Series II, Cambridge Univ. Press, New York, 1974.

[Ma4] _ Geometry of iterated loop spaces, Lecture Notes in Math., vol. 271, Springer-Verlag, Berlin and New York, 1972.

[Mo1] T. Matumoto, On G-CW complexes and a theorem of J. H. C. Whitehead, J. Fac. Sci. Univ. Tokyo Sect. IA Math. 18 (1971/72), 363-374.

[MHW] J. P. May, H. Hauschild and S. Waner, Equivariant infinite loop spaces (in preparation).

[St1] J. D. Stasheff, A classification theorem for fiber spaces, Topology 2 (1963), 239-246.

[Wa1] S. Waner, Cyclic group actions and the Adams conjecture (to appear).

[Wa2] _ Equivariant homotopy theory and Milnor's Theorem, Trans. Amer. Math. Soc. 258 (1980), $351-368$.

[Wæ3] __, Equivariant fibrations and transfer, Trans. Amer. Math. Soc. 258 (1980), 369-384.

Department of Mathematics, Princeton University, Princeton, New Jersey 08540 